\title{
Musculoskeletal ultrasound guided manoeuvres - security profile
}

\author{
Mihaela C. Micu1, Violeta M. Vlad², Sorana D. Bolboacă${ }^{3}$, Mihaela Cârlig ${ }^{4}$, György I. Bodizs $^{5}$, \\ Alina G. Duţu' ${ }^{6}$, Daniela Fodor ${ }^{7}$
}

${ }^{1}$ Rheumatology Division, Rehabilitation Clinical Hospital Cluj-Napoca, ${ }^{2}$ Rheumatology Department, "Sfânta Maria" Hospital, Bucharest, ${ }^{3}$ Department of Medical Informatics and Biostatistics, "Iuliu Hatieganu" University of Medicine and Pharmacy Cluj-Napoca, ${ }^{4}$ University Medical and Surgical Center Interservisan, Cluj-Napoca, ${ }^{5}$ Laboratory Unit, Rehabilitation Clinical Hospital Cluj-Napoca, ${ }^{6}$ Biochemistry Department, "Iuliu Hatieganu" University of Medicine and Pharmacy Cluj-Napoca, ${ }^{7} 2^{\text {nd }}$ Internal Medicine Department, "Iuliu Hatieganu" University of Medicine and Pharmacy Cluj-Napoca, Romania

\begin{abstract}
Background: In daily rheumatology clinical practice, routine interventional musculoskeletal ultrasound (MSUS) guided maneuvers such as aspiration, intraarticular or periarticular drug injections require efficient cleaning and disinfection methods for both transducer and patient's skin. Aim: To study the efficacy of probe and skin disinfection measures after using simple protocols, to identify the prevalence of septic and other drug related side effects after MSUS guided interventions and to quantify the total procedure time. Material and methods: Recruitment of consecutive patients with different joint/ periarticular MSUS guided interventions was made in 3 medical centers. Bacterial load was determined on the transducers footprint after dry cleaning with the removal of any gel trace and on patient's skin after rigorous skin disinfection with either Bethadine or alcohol $70^{\circ}$ and Bethadine. Non-sterile gel was used as an ultrasound transmission medium. The time spent for some of the invasive procedures was quantified. Results: Nine hundred and ninety eight MSUS guided interventional maneuvers were performed in 945 patients with inflammatory and degenerative musculoskeletal pathologies. Staphylococcus epidermidis was identified in $13.33 \%$ cases of the skin bacterial load analysis and in $37.50 \%$ cases of the footprint analysis. In two patients pathogenetic germs were detected on the skin. No septic post-procedural complications were reported. In $0.6 \%$ of the cohort other side effects occurred: aseptic osteonecrosis, skin depigmentation at injection site and iatrogenic microcristaline reactions. The median time frame dedicated to the intervention was 6 minutes. Conclusion: Rigorous transducer dry cleaning and Bethadine/ Bethadine and alcohol $70^{\circ}$ skin disinfection are efficacious methods. The risk for septic complications and other drug related side effects related to MSUS guided injections is very low in this context. A correct injection technique must accompany the previous requests. Rapid and safe interventional maneuvers reduce the risks and control the costs of the healthcare system.
\end{abstract}

Keywords: musculoskeletal ultrasound, interventional, disinfection, septic complications, side effects

\section{Introduction}

Interventional musculoskeletal ultrasound (MSUS) guided manoeuvres refer to a large category of percuta-

Received 05.06.2014 Accepted 01.07.2014

Med Ultrason

2014, Vol. 16, No 3, 214-221

Corresponding author: Micu Mihaela Cosmina

Rheumatology Division,

Clinical Rehabilitation Hospital,

46-50 Viilor street

400306, Cluj- Napoca, Romania

Phone: +40264207021, Fax: +40264453131

E-mail:mcmicu@yahoo.com neous invasive procedures the using ultrasound examination technique for needle guidance. In daily rheumatology clinical practice, several manoeuvres are more frequently performed such as aspiration or drug injection in joints, tendon sheaths and periarticular structures, or perineural anaesthetic blocks. More rarely, complex manoeuvres such as biopsies, foreign body extraction, or rotator cuff calcifications needling and aspiration techniques are done [1-5].

Over the last decade, MSUS guided manoeuvres have gained higher interest in clinical practice. Consistent published medical data assign higher drug deposition accuracy and higher efficacy in comparison to blind ma- 
noeuvres along with better procedural and postprocedural pain and functional outcome [6-20]. All these advantages together with the possibility to perform interventional manoeuvres quickly and safely, immediately after the MSUS evaluation, in the same room and by the same physician, using a non-radiant imaging tool, generate an important economic impact on the healthcare system due to the direct and indirect cost savings [16,21].

Still, there is an ongoing debate concerning the security profile of MSUS guided manoeuvres when using simple transducer and skin disinfection protocols. Repetitive use of the same transducer in different anatomic areas or between different patients, the small distance between the transducer and injection site raises the question for the true risk of septic complications when performing these kinds of manoeuvres [22-26]. On the other hand, sophisticated disinfection protocols are time consuming and expensive and, at the end, may limit the number of performing physicians. Another important aspect is related to drug side effects: aseptic osteonecrosis of the injected joints, local skin depigmentation or iatrogenic microcristaline synovitis/ tenosynovitis after corticosteroid drugs (CS) injection, etc., all these events being described as possible side effects in the literature [5,20,27-30].

Therefore, the aims of our study were to perform a prospective study with the focus on probe and skin disinfection efficacy after using simple protocols, to identify the prevalence of septic and other drug related side effects after MSUS guided interventional manoeuvres, and to estimate the total procedure duration.

\section{Material and method}

Recruitment of consecutive patients with different joint/ periarticular pathologies was made in three medical centres: Rheumatology Division, Rehabilitation Clinical Hospital Cluj-Napoca, Rheumatology Department, "Sfânta Maria" Hospital, Bucharest, and 2nd Internal Medicine Department, "Iuliu Hatieganu" University of Medicine and Pharmacy Cluj-Napoca, Romania (randomly named medical centre 1, 2, and 3) between February 2013- January 2014. Clinical and MSUS evaluation was performed by 3 rheumatologists (MCM 7 years/ VV 10 years/DF 15 years of experience in MSUS), with Esaote MyLab 50/70 ultrasound machines, according to current guidelines and protocols for each anatomic region [31]. All MSUS guided interventional manoeuvres performed in this time frame were recorded. The decision for a certain interventional manoeuvre and timing related to other therapeutic modalities addressed to the patient was made by each performing physician for their own

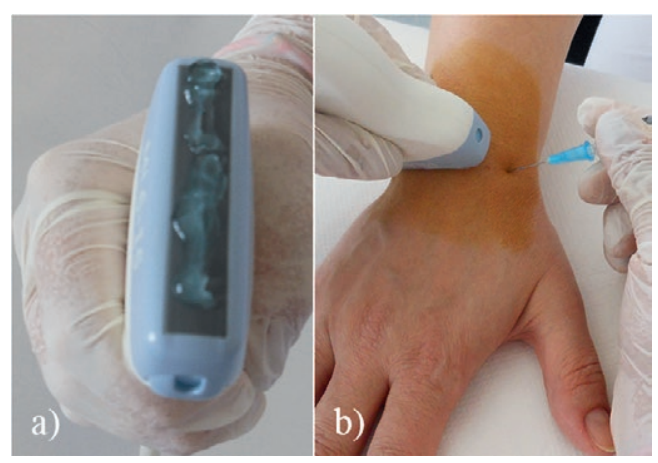

Fig 1. a) Transducers footprint is covered by a small amount of non-sterile gel while preparing for injection; b) Transducer is placed on the disinfected skin; the total amount of gel remains between the footprint and skin. Security distance between the needle and transducer is kept.

group of patients. The patients signed a written consent, in agreement with the declaration of Helsinki, obligatory in our medical units, prior to any investigation or interventional manoeuvre. In addition, the consent specifies the possibility of clinical data use for scientific purposes. The local Ethics Committee approved the study.

The preparation for the performing physician included hand washing, gloves, mask and robe. No sterile cover for the probe was used, neither sterile gel. Non-sterile gel was placed strictly under the probes footprint (fig 1).

The standard preparation of the probe follows a protocol in which dry soft paper cleaning is performed before the first session, with the first patient, and after each examined anatomic area (in order to clean any trace of the remaining gel) and before performing the next examination or interventional manoeuvre in the same patient. Two bacterial loads were evaluated: first from the footprint surface after the cleaning protocol was applied and second from on the patient's skin after disinfection protocol was done

Examination of the transducers bacterial load was made by touching the footprint surface with a previously humidified (sterile saline solution) cotton swab. The cotton swab was kept for 10-15 minutes in $9 \mathrm{ml}$ sterile saline peptone followed by insemination in two different culture mediums: blood agar and Bromothymol-blue lactose agar (AABTL). The culture technique underwent a standard protocol with incubation for 48 hours at $37^{\circ} \mathrm{C}$. In cases where bacterial colonies were detected, the following supplementary tests were performed: chatalase test, gram stain, coagulase test, Chapman medium insemination, and Novobiocine test.

The second bacterial load determination was made from the patient's skin exactly before executing the cho- 
sen interventional manoeuvre. After skin disinfection with alcohol $70^{\circ}$ followed by Bethadine (medical centre 2) or only Bethadine (medical centrel and 3) a sterile saline serum humidified cotton swab touched an area of $4 \mathrm{~cm}^{2}$ in the proximity of the transducers footprint. Afterwards, the invasive procedure was performed and at the end the transducer underwent dry cleaning again.

Evaluation of the time spent for performing the interventional manoeuvre was monitored in 87 patients with different pathologies. The time spent comprised the dry cleaning of the probe, hands wash and gloves on, disinfection of skin, placement of the probe in order to expose optimally the target lesion, insertion of the needle, aspiration, drug injection, needle retraction, disinfection and haemostasis if necessary.

Each patient was instructed to report in the first 48$72 \mathrm{~h}$ after injection of the occurrence of systemic or local symptoms and signs of infection (redness, swelling, pain, fever, etc.) and to return for follow up after 3 weeks or at any moment if any suspicion of side effects related to the procedure were detected. No bed rest indications were made after the procedure, with the exception of a subset of patients with ongoing anticoagulant therapy.

\section{Statistic analyses}

Data were analyzed according to the type of variables. Qualitative data were summarized as percentage and associated 95\% confidence interval (provided in squared brackets along the manuscript), confidence interval computed using an exact approach [32]. Quantitative data were summarized as median and interquartile range (provided in round brackets along the manuscript) whenever data proved not to follow a normal distribution. Comparisons between two groups were done with $\mathrm{Z}$ test for proportions when qualitative data were of interest or Mann-Whitney test for quantitative data proved not to follow a normal distribution. Statistical analysis was conducted with the Statistica (v.8) program at a significance level of $5 \%$. Any $\mathrm{p}<0.05$ was considered statistically significant. Graphical representations were done using Microsoft Excel.

\section{Results}

Enrolment of 945 consecutive patients totalizing 998 interventional MSUS guided manoeuvres was made. Demographic data, disease spectrum and activity scores for rheumatoid arthritis (RA) and ankylosing spondylitis (AS) are presented in Table I. A significantly higher proportion of investigated subjects were women (women: 66.46\% [63.39-69.42]; men: 33.54\% [30.58-36.61]; Zstatistics $=-21.43, \mathrm{p}<0.0001)$.

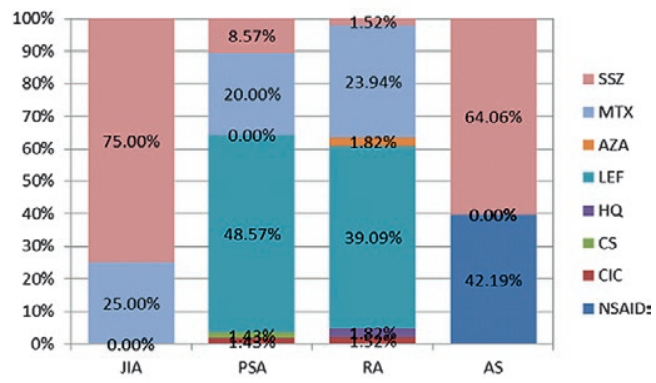

Fig 2. Distribution of different therapies in juvenile idiopathic arthritis (JIA), psoriatic arthritis (PSA), rheumatoid arthritis (RA), and ankylosing spondylitis (AS). SSZ- Salazophyrine, MTX- Metotrexate, AZA- azathioprine, LEF- Leflunomide, HQ- Hydroxiclorochine, CS- corticosteroids, CIC-cyclosporine, NSAIDs- nonsteroidal antiinflammatory drugs.

Table I. Characteristics of the patients group (number of the patients- 945)

\begin{tabular}{lcccc}
\hline Diagnosis & $\mathbf{N}$ & Age (years) & Disease onset (years) & Disease activity \\
\hline JIA & 4 & $10(9.75-11.25)$ & $3.5(3-4)$ & n.a. \\
PSA & 70 & $60(55-66.75)$ & $6(5-8)$ & n.a. \\
Reactive arthritis & 4 & $33.5(31.75-37.25)$ & $8(7-8)$ & n.a. \\
Carpal tunnel syndrome & 16 & $56(54-65)$ & n.a. & n.a. \\
Degenerative lesions* & 256 & $58.5(61.0-67)$ & n.a. & n.a. \\
Gout & 47 & $63(54-69)$ & $3.5(1.5-4)$ & n.a. \\
OA & 132 & $63(54-69)$ & n.a. & n.a. \\
RA & 330 & $63(55-70)$ & $5(3.5-7)$ & $4.03(3.7-5.12)$ \\
AS & 64 & $40(31.25-48)$ & $4(3-6.5)$ & $4.70(3.99-6.2)$ \\
Trauma & 22 & $20(18-26)$ & n.a. & n.a. \\
\hline
\end{tabular}

Results are expressed in median (Q1-Q3) (lower and upper quartiles). Disease activity score in RA was expressed as DAS 28 (CRP). Disease activity score in AS was expressed as BASDAI. N- number of patients, *-periarticular structures, JIA- juvenile idiopathic arthritis, PSApsoriatic arthritis, OA- osteoarthritis, RA- rheumatoid arthritis, AS- ankylosing spondylitis. 


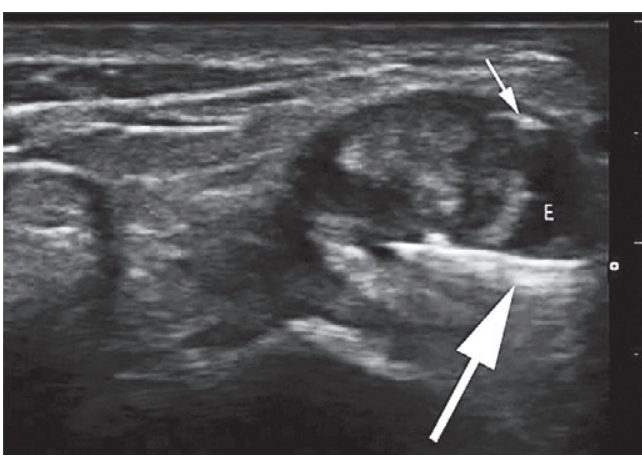

Fig 3. Free hand technique injection with corticosteroids (CS) inside the tendon sheath at the level of the first extensors compartment. Needle is identified penetrating the tendon sheath (big white arrow), CS antigravitational accumulation after injection (small white arrow), E- effusion inside the tendon sheath.

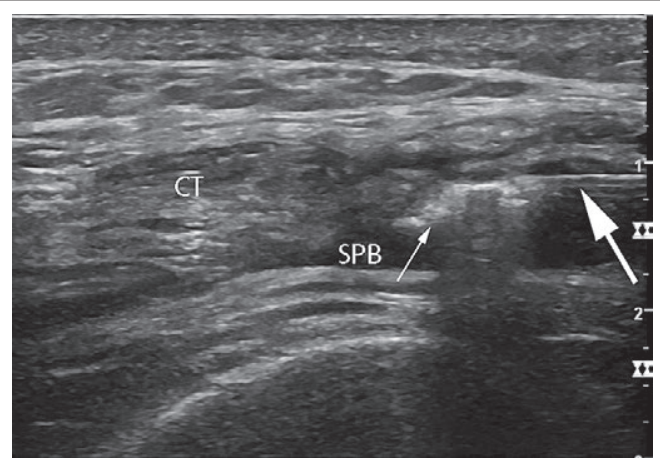

Fig 4. Free hand technique injection with Hyaluronic acid preparate at knee level. The needle is visualized (big white arrow), the drug is identified as a hyperechoic mass (small white arrow), SPBsuprapatellar bursa, CT- quadriceps tendon.

Table II. Number, percentage, and type of injected areas

\begin{tabular}{|c|c|c|c|c|c|c|}
\hline \multirow{2}{*}{ Injected area } & \multirow{2}{*}{ CS } & \multirow{2}{*}{ AO } & \multirow{2}{*}{ HA } & \multirow{2}{*}{ Total } & \multicolumn{2}{|c|}{$95 \% \mathrm{CI}$} \\
\hline & & & & & Lower Bound & Upper Bound \\
\hline $\mathrm{AC}$ & 3 & 0 & 1 & $0.40 \%$ & 0.10 & 1.00 \\
\hline SASDB & 276 & 0 & 0 & $27.65 \%$ & 24.75 & 31.06 \\
\hline $\mathrm{GH}$ & 3 & 0 & 0 & $0.30 \%$ & 0.10 & 0.90 \\
\hline Elbow joint & 16 & 0 & 4 & $2.00 \%$ & 1.20 & 3.11 \\
\hline Elbow enthesis & 102 & 0 & 0 & $10.22 \%$ & 8.42 & 12.22 \\
\hline $\mathrm{RC}, \mathrm{IC}$ & 12 & 0 & 0 & $1.20 \%$ & 0.50 & 2.60 \\
\hline CTS & 19 & 0 & 0 & $1.90 \%$ & 1.10 & 2.91 \\
\hline MCP & 5 & 0 & 0 & $0.50 \%$ & 0.20 & 1.20 \\
\hline DIP & 1 & 0 & 0 & $0.10 \%$ & 0.00 & 0.60 \\
\hline Knee joint & 224 & 0 & 17 & $24.15 \%$ & 21.54 & 26.95 \\
\hline Popliteal cyst & 23 & 0 & 0 & $2.30 \%$ & 1.50 & 3.41 \\
\hline $\mathrm{CF}$ & 26 & 0 & 25 & $5.11 \%$ & 3.81 & 6.61 \\
\hline PT & 32 & 0 & 0 & $3.21 \%$ & 2.20 & 4.51 \\
\hline $\mathrm{TT}$ & 8 & 0 & 8 & $1.60 \%$ & 0.90 & 2.61 \\
\hline Subtalar joints & 2 & 0 & 0 & $0.20 \%$ & 0.00 & 0.70 \\
\hline Plantar fascia & 35 & 0 & 0 & $3.51 \%$ & 2.40 & 4.81 \\
\hline Achille's bursa & 11 & 0 & 0 & $1.10 \%$ & 0.50 & 2.00 \\
\hline Olecranon bursa & 16 & 0 & 0 & $1.60 \%$ & 0.90 & 2.61 \\
\hline MTP & 15 & 0 & 6 & $2.10 \%$ & 1.30 & 3.21 \\
\hline Trauma & 0 & 43 & 0 & $4.31 \%$ & 3.11 & 5.71 \\
\hline Tenosynovitis & 65 & 0 & 0 & $6.51 \%$ & 5.01 & 8.22 \\
\hline Total & 894 & 43 & 61 & $100 \%$ & & \\
\hline
\end{tabular}

CS- corticosteroid;AO- Aspiration only; HA- hyaluronic acid; AC- acromio-clavicular joint; SASDB- subacromial subdeltoid bursa; GH- glenohumeral joint; RC- radiocarpal joint; IC- intercarpal joint; CTS- carpal tunnel syndrome; MCP- metacarpophalangeal joint; DIP- distal interphalangeal joint, CF- coxofemoral joint; PT- Peritrochanterian; TT- tibiotalar joint; MTP- metatarsophalangeal joint. 
In the patients group, 468 subjects were diagnosed with RA, AS, psoriatic arthritis (PSA), and juvenile idiopathic arthritis (JIA). Out of this subset, 358 subjects received synthetic DMARD monotherapy $(76.50 \%$ [72.44-80.13]) and 110 subjects received combined therapy with synthetic DMARDs or synthetic DMARD and biologic therapy $(23.50 \%$ [21.50-28.54]). A significantly higher percentage of subjects was treated with monotherapy (Z-statistics $=-25.48, \mathrm{p}<0.0001)$ (fig 2). Inside the subgroup with chronic inflammatory diseases, only $8 \%$ of the patients (totalizing 38 patients) were in remission status at the time of recruitment.

Overall the local injected medication was represented by CS (Bethamethasone, 89.58\% [87.47-91.38]), followed by viscosupplementation (Hyaluronic acids derivates, HA) (6.11\% [4.71-7.82]) (fig 3, fig 4); effusion evacuation was present in $4.31 \%$ [3.11-5.71] of the cases. Table II presents the percentage, type of injected anatomical areas and medication used.

Skin bacterial load determinations were performed randomly in 45 of analyzed subjects. The examinations were made as follows: in medical centre 1-24 determinations in 7 different days, different anatomical regions: knee, hands, shoulder, and ankle and in medical centre 2-21 determinations in 4 different days in following anatomic regions: knee, plantar fascia, shoulder, hand, and ankle. In $13.33 \%$ [4.49-26.62] of the cases bacterial growth was identified. Four subjects were detected with staphylococcus epidermidis, 1 case with bacillus cereus and 1 case with staphylococcus aureus (only in medical centre 2). No septic complications were reported after the interventional maneuvers in these 45 patients.

Transducers footprint bacterial load was determined in 16 cases (medical centre 2-10 determinations in 4 different days and medical centre 1-6 determinations in the same day). Six cases with staphylococcus epidermidis representing $37.50 \%$ [12.89-62.11] were detected, (only in medical centre 1). No septic complications were recorded post- interventional in these 16 patients.

Overall, out of 945 patients, in $99.40 \%$ [98.70-99.80] of the cases no post-interventional complications occurred. In 2 subjects which underwent coxofemoral joint CS injection, aseptic hip osteonecrosis was diagnosed 6 months later on MRI, in 2 subjects iatrogenic microcristaline reaction after Achilles bursa CS injection was identified in the first 24 hours after injection (MSUS repeated) and in 1 subject skin depigmentation at injection site after CS occurred. One patient who received viscosupplementation drug deposition at the level of the lateral recess of the knee experienced an intense unexplained knee pain after 3 weeks (event announced by phone call). No patient presented post-procedural septic complications.
The median time frame dedicated to the intervention was of 6 minutes with an interquartile range from 5 to 7 minutes.

\section{Discussions}

MSUS is a valuable tool for the physicians allowing real time visual assistance, helping them to perform quickly (immediately after the first consultation, at bedside sometimes), safely and precisely the interventional manoeuvres. The method is even more helpful in cases with anatomic variations - congenital or postsurgical because it enables the physician to see the area of interest, to optimize the interventional manoeuvre per se, subsequently the patients outcome, and to minimize the risk of complications [21,33-40].

When inappropriate cleaning protocols are used, probe and ultrasound transmission gel used in daily practice can be a source of bacterial transmission among patients. By now, there is no consensus regarding these protocols for rheumatology settings. Reports of infectious diseases transmitted via probe or associated with the use of transmission gel occur more frequently in surgical healthcare settings, in patients with unhealed wounds, burns and those in intensive care units $[24,25,41,42]$. Some recent general guidelines which include the obligation of using sterile gel were proposed in order to minimize the infection risk [43]. Despite these protection measures, it has been shown that transmission gel can be contaminated (from manufacturer) leading to infections. Apparently, bacteriostatic components as parabens or methyl benzoate are not always a warranty for a high sterility standard as long as staphylococcus aureus, pseudomonas aeruginosa, and E. Coli could be identified in 'sterile' transmission gel [43-46].

In our daily practice, our protocol does not include the use of sterile gel. We consider that this measure is not necessary when probe and skin disinfection are properly done and security distance between needle and probe is kept during the entire interventional procedure.

In rheumatologic clinical practice patients have more rarely major skin problems such as extended wounds or other lesions with infection potential. Still, a higher risk for post-procedural infections could be linked to the patient's immunosuppressive status due to chronic inflammatory disease and/or systemic medication. Indeed, multiple MSUS joint/periarticular evaluations/interventions in the same patient may enhance the iatrogenic infection risk [43]. In our study we show that no septic event occurred in any of the patients, independently of the underlying pathology, disease activity status, immunosuppressive therapeutic strategy or repetitive MSUS guided 
interventions in the same patient. In fact, in the entire patients group, more than $50 \%$ were diagnosed with chronic inflammatory diseases out of which approximately $75 \%$ were treated with synthetic DMARD monotherapy and the rest with different drug combinations. Only 38 patients representing $8 \%$ of the subset were in remission status when data were registered. These results are in line with the study of Cervini et al that showed a very low incidence of serious septic events (14 cases) after different types of US guided interventions performed in over 13,000 patients with various pathologies [47].

It is well known that repetitive, aggressive (alcoholic solutions) disinfection of the probe produces irreversible damage translated into impaired ultrasound images $[25,26]$. Therefore, efficient but safe (non-alcoholic) probe cleaning and skin disinfection protocols together with a correct injection technique which implies a security distance of minimum $0.5 \mathrm{~cm}$ between the footprint and injection site are mandatory in avoiding septic side effects. Our objective was to ascertain whether the current protocol for probes cleaning and skin disinfection in our units is adequate to prevent cross contamination. Our data show that removal of any visible gel trace by using a simple dry cleaning method of the probe ensures a safe interventional procedure with no septic side effects, results confirmed also by other recent studies $[22,42]$. Indeed, rigorous skin disinfection with both described alternatives assures safe injection conditions. In our patients, the bacterial load was determined on the skin by checking an area of 4 square $\mathrm{cm}$ but in reality the needle tip touches an area of less than $1 \mathrm{~mm}^{2}$ when penetrating the skin. Ubiquitous bacteria such as staphylococcus epidermidis may be present on patients' skin or on the transducers' footprint but does not represent a true risk factor for further infectious events as proved in our study.

In fact, in one of our centres, a higher incidence of staphylococcus epidermidis bacterial load was detected on the transducers footprint. A potential explanation for this finding could be that patients were evaluated with the same probe not only for musculoskeletal disorders but also for other different pathologies. The probe was placed in the same day on abdominal, pelvic, axillary or neck region skin and this could contribute to a higher incidence of staphylococcus epidermidis detection. Moreover, none of the 2 patients in which we identified pathogenetic bacterial skin load (bacillus cereus and staphylococcus aureus) developed infectious complications after the interventional manoeuvre. In this last example, two explanations could be valid: the needle penetrated in a sterile area inside those $4 \mathrm{~cm}^{2}$ of skin or the skin bacterial load was too low to trigger an infectious event.
Apart from the infectious risk, current literature cite also other rare side effects due to the injected intra/paraarticular drugs such as aseptic osteonecrosis after CS intra-articular injection, intraarticular granulomatosus inflammation developed after viscosupplementation with HA, secondary iatrogenic crystalline synovitis, tendon ruptures, or more mild local effects as skin depigmentation, with higher prevalence in immunodepressed patients $[28,29]$. In our cohort, we reported 2 patients with possible aseptic hip osteonecrosis related to CS injection diagnosed 6 months later, 2 cases of iatrogenic microcristaline bursitis and one case of local skin depigmentation, totalizing $0.05 \%$ of the interventional manoeuvres. A 100\% link between the hip aseptic osteonecrosis and the intraarticular CS injection could not be demonstrated with certitude. The 2 patients were diagnosed with hip coxitis refractory to systemic NSAIDs that why CS and hip intra-articular CS injection was decided. No baseline MRI was performed, therefore primary aseptic osteonecrosis could not be ruled out based only on MSUS evaluation. Hip effusion may be part of this of the underling pathology.

In clinical practice, MSUS guided injection technique has to show advantages over classic ones: better efficacy, a comparable or a better safety profile together with a similar or higher, if possible, procedure speed. MSUS guided invasive manoeuvres offer the possibility to see the target structure and surrounding tissues, to access millimetric spaces, to monitor the entire invasive procedure or to repeat the evaluation if necessary. But the unanswered question is: are these MSUS guided interventional manoeuvres feasible for clinical practice? This equation implies also the calculation of the absolute time spent for performing MSUS guided interventions [21,48]. Usually, less experienced doctors in performing MSUS guided manoeuvres are discouraged by complicated disinfection protocols, sophisticated equipment including sterile guiding systems, sterile gel, more time spent with the patient, and more costs. In our study we show that after MSUS target region evaluation, followed by a simple but efficient disinfection protocol, a reasonable timeframe totalizing maximum 7 minutes was necessary to accomplish the procedure. As far as we know, this is the first study which includes data regarding time evaluation when performing different MSUS interventional manoeuvres.

The main limit of our study is the low number of probe and skin bacterial load determinations in comparison to the total amount of interventional procedures (and sometimes data was collected in one single day). Another limit would be the absence of comparison with classic landmark guided injections. 
In conclusion, MSUS guided manoeuvres are ideal for clinical practice because they are not only highly efficient but also safe, rapid, and cheap. The incidence for septic post-procedural events was zero in our study along with a very low risk for other types of complications.

The capacity to improve the patients' outcome, to reduce the procedural risk and to control the costs due to significant cost savings related to the increase use of invasive procedures in the outpatient clinic has in the end an important impact on health care resources.

Acknowledgments: We wish to thank to Maria Mureșan, Mihaela Costişor, Claudia Ciorca (Rehabilitation Clinical Hospital, Cluj-Napoca) for their valuable contribution in performing this study.

\section{Conflict of interest: none}

\section{References}

1. Karim Z, Wakefield RJ, Conaghan PG, et al. The impact of ultrasonography on diagnosis and management of patients with musculoskeletal conditions. Arthritis Rheum 2000; 44: 2932-2933.

2. D’Agostino MA, Ayral X, Baron G, Ravaud P, Breban M, Dougados M. Impact of ultrasound imaging on local corticosteroid injections of symptomatic ankle, hind-,and midfoot in chronic inflammatory diseases. Arthritis Rheum 2005; 53: 284-292.

3. Micu MC, Alcalde M, Saenz JI, et al. Impact of musculoskeletal ultrasound in an outpatient rheumatology clinic. Arthritis Care Res 2013; 65: 615-621.

4. Davidson J, Jayaraman S. Guided interventions in musculoskeletal ultrasound: what's the evidence? Clin Radiol 2011; 66: 140-152.

5. Epis O, Bruschi E. Interventional ultrasound: a critical overview on ultrasound- guided injections and biopsies. Clin Exp Rheumatol 2014; 32 (Suppl 80): S78-S84.

6. Komppa GH, Northern JR Sr, Haas DK, Lisecki E, Ghaed N. Ultrasound guidance for needle aspiration of the hip in patients with painful hip prosthesis. J Clin Ultrasound 1985; 13: 433-434.

7. Jones A, Regan M, Ledingham J, Pattrick M, Manhire A, Doherty M. Importance of placement of intra-articular steroid injections. BMJ 1993; 307: 1329-1330.

8. Eustace JA, Brophy DP, Gibney RP, Bresnihan B, FitzGerald $\mathrm{O}$. Comparison of the accuracy of steroid placement with clinical outcome in patients with shoulder symptoms. Ann Rheum Dis 1997; 56: 59-63.

9. Zingas C, Failla JM, Van Holsbeeck M. Injection accuracy and clinical relief of de Quervain's tendonitis. J Hand Surg 1998; 23: 89-96.

10. Grassi W, Lamanna G, Farina A, Cervini C. Synovitis of small joints sonographyc guided diagnostic and therapeutic approach. Ann Rheum Dis 1999; 58: 595-597.
11. Koski JM. Ultrasound guided injections in rheumatology. J Rheumatol 2000; 27: 2131-2138.

12. Sethi PM, El Attrache N. Accuracy of intra- articular injection of the gleno- humeral joint: A cadaveric study. Orthopedics 2006; 29: 149-152.

13. Lohman M, Vasenius J, Nieminen O. Ultrasound guidance for puncture and injection of radiocarpal joint. Acta Radiol 2007; 48: 744-747.

14. Lopes RV, Furtado RNV, Parmigiani L, Rosenfeld A, Fernandes AR, Natour J. Accuracy of intra-articular injections in peripheral joints performed blindly in patients with rheumatoid arthritis. Rheumatology (Oxford) 2008; 47: 17921794.

15. Cunnington J, Marshall N, Hide G, et al. A randomized, double - blind, controlled study of ultrasound-guided corticosteroid injection into the joint of patients with inflammatory arthritis. Arthritis Rheum 2010; 63: 1862-1869.

16. Berkoff DJ, Miller LE, Block JE. Clinical utility of ultrasound guidance for intra-articular knee injections: a review. Clin Interv Aging 2012; 7:89-95.

17. Iagnocco A, Naredo E. Ultrasound-guided corticosteroid injection in rheumatology: accuracy or efficacy? Rheumatology (Oxford) 2010; 49: 1427-1428.

18. Balint PV, Kane D, Hunter J, McInnes IB, Field M, Sturrock RD. Ultrasound guided versus conventional joint and soft tissue fluid aspiration in rheumatology practice: a pilot study. J Rheumatol 2002; 29: 2209-2213.

19. D'Agostino MA, Schmidt WA. Ultrasound-guided injections in rheumatology: Actual knowledge on efficacy and procedures. Best Pract Res Clin Rheumatol 2013; 27: 283294.

20. Epis O, Iagnocco A, Meenagh G, et al. Ultrasound imaging for the rheumatologist XVI. Ultrasound - guided procedures. Clin Exp Rheumatol 2008; 26: 515-518.

21. Slawsky K, McInnis M, Goss TF, Lee DW. The clinical economics of ultrasound- guided procedures. Technical Report. General Electric, Wauwatosa, WI, 2011.

22. Muradali D, Gold WL, Phillips A, Wilson S. Can ultrasound probes and coupling gel be a source of nosocomial infectionin patients undergoing sonography? An in vivo and in vitro study. AJR Am J Roentgenol 1995; 164: 15211524.

23. Tesch C, Froschle G. Sonography machines as a source of infection. AJR Am J Roentgenol 1997; 168: 567-568.

24. Hayashi S, Koibuchi H, Taniguchi N, Hirai Y. Evaluation of procedures for decontaminating ultrasound probes. J Med Ultrasonics 2012; 39: 11-14.

25. Koibuchi H, Kotani K, Taniguchi N. Ultrasound probes as a possible vector of bacterial transmission. Med Ultrason 2013; 15: 41-44.

26. Koibuchi H, Fujii Y, Kotani K, et al. Degradation of ultrasound probes caused by disinfection with alcohol. J Med Ultrasonics 2011; 38: 97-100.

27. Migliore A, Tormenta S, Martin Martin LS, et al. Safety profile of 185 ultrasound guided intra-articular injections for treatment of rheumatic diseases in the hip. Reumatismo 2004; 56: 104-109. 
28. Kruse DW. Intraarticular cortisone injection for osteoarthritis of the hip. Is it effective? Is it safe? Curr Rev Musculoskeletal Med 2008; 1: 227-233.

29. Migliore A, Giovannangeli F, Granata M, Lagana B (2010). Hyalgan g-f 20: review of its safety and efficacy in the management of joint pain in osteoarthritis. Clin Med Insights Arthritis Musculoskeletal Disord 2010; 3: 55-68.

30. Nolsoe C, Nielsen L, Torp-Pedersen S, Holm HH. Major complications and deaths due to interventional ultrasonography: A review of 8000 cases. J Clin Ultrasound 1990; 18: 179-184.

31. Backhaus M, Burmester G, Gerber T, et al. Guidelines for musculoskeletal ultrasound in rheumatology. Ann Rheum Dis 2001; 60:641-649.

32. Jäntschi L, Bolboacă SD. Exact probabilities and confidence limits for binomial samples: applied to the difference between two proportions. ScientificWorldJournal 2010; 10: 865-878.

33. Raza K, Lee CY, Pilling D, et al. Ultrasound guidance allows needle placement and aspiration from small joints in patients with early inflammatory arthritis. Rheumatology (Oxford) 2003; 42: 976-979.

34. Taras JS, Raphael JS, Pan WT, Movagharnia F, Sotereanos DG. Corticosteroid injections for trigger digits : Is intrasheat injection necessary? J Hand Surg Am 1998; 23: 717-722.

35. Sibbitt WL Jr, Peisajovich A, Michael AA, et al. Does sonographyc needle guidance affect the clinical outcome of intraarticular injections? J Rheumatol 2009; 36: 1892-1902.

36. Fodor D (eds). Aplicatii ale ecografiei musculoscheletale in reumatologie. Ed. Medicală, Bucureşti, 2013.

37. Naredo E, Cabero F, Cruz A, Uson J, Palop MJ, Crespo M. Ultrasound guided musculoskeletal injections. Ann Rheum Dis 2005; 64: 341 .

38. Naredo E, Cabero F, Beneyto P, et al. A randomized comparative study of short term response to blind injection versus sonographic-guided injection of local corticosteroids in patients with painful shoulder. J Rheumatol 2004; 31: 308314.
39. Bruyn GA, Schmidt WA. How to perform ultrasound guided injections. Best Pract Res Clin Rheumatol 2009; 23: 269-279.

40. McAlindon T, Kissin E, Nazarian L, et al. American College of Rheumatology report on reasonable use of musculoskeletal ultrasonography in rheumatology clinical practice. Arthritis Care Res (Hoboken) 2012; 64: 1625-1640.

41. Mirza WA, Imam HS, Kharal MS, et al. Cleaning methods for ultrasound probes. J Coll Physicians Surg Pak 2008; 18: 286-289.

42. Bello TO, Taiwo SS, Oparinde DP, Hassan WO, Amure JO. Risk of nosocomial bacteria transmission: evaluation of cleaning methods of probes used for routine ultrasonography. West Afr J Med 2005; 24: 167-170.

43. Oleszkowicz SC, Chittick P, Russo V, Keller P, Sims M, Band J. Infections associated with use of ultrasound transmission gel: proposed guidelines to minimize risk. Infect Control Hosp Epidemiol 2012; 33: 1235-1237.

44. Hutchinson J, Runge W, Mulvey M, et al. Burkholderia cepacia infections associated with intrinsically contaminated ultrasound gel: the role of microbial degradation of parabens. Infect Control Hosp Epidemiol 2004; 25: 291-296.

45. Weist K, Wendt C, Petersen LR, Versmold H, Ruden H. An outbreak of pyodermas among neonates caused by ultrasound gel contaminated with methicillin-susceptible Staphylococcus aureus. Infect Control Hosp Epidemiol 2000; 21 : 761-764.

46. Gaillot O, Maruejouls C, Abachin E, et al. Nosocomial outbreak of Klebsiella pneumoniae producing SHV-5 extended-spectrum $\beta$-lactamase, originating from a contaminated ultrasonography coupling gel. J Clin Microbiol 1998; 36: 1357-1360.

47. Cervini P, Hesley GK, Thompson RL, Sampathkumar P, Knudsen JM. Incidence of infectious complications after an ultrasound- guided intervention. AJR Am J Roentgenol 2010; 195: 846-850.

48. Micu MC, Iagnocco A. Musculoskeletal ultrasound guiding shoulder injection when "one picture is worth ten thousand landmarks". Med Ultrason 2013; 15: 3-5. 